\title{
Stability to a Kind of Functional Differential Equations of Second Order with Multiple Delays by Fixed Points
}

\author{
Cemil Tunç ${ }^{1}$ and Emel Biçer ${ }^{2}$ \\ ${ }^{1}$ Department of Mathematics, Faculty of Sciences, Yüzüncü Yıl University, 65080 Van, Turkey \\ ${ }^{2}$ Department of Mathematics, Faculty of Arts and Sciences, Bingöl University, 12000 Bingöl, Turkey \\ Correspondence should be addressed to Emel Biçer; ekayabicer@gmail.com
}

Received 13 January 2014; Revised 29 March 2014; Accepted 30 March 2014; Published 17 April 2014

Academic Editor: Samir Saker

Copyright @ 2014 C. Tunç and E. Biçer. This is an open access article distributed under the Creative Commons Attribution License, which permits unrestricted use, distribution, and reproduction in any medium, provided the original work is properly cited.

We discuss the stability of solutions to a kind of scalar Liénard type equations with multiple variable delays by means of the fixed point technique under an exponentially weighted metric. By this work, we improve some related results from one delay to multiple variable delays.

\section{Introduction}

For more than one hundred years, Lyapunov's direct (second) method has been very effectively used to investigate the stability problems in ordinary and functional differential equations. This method is one of the highly effective methods to determine the stability properties of solutions of ordinary and functional differential equations of higher order in the literature. However, till now, constructing or defining Lyapunov functions or functionals which give a meaningful discussion remains a general problem in the literature. In recent years, many researchers discussed that the fixed point theory has an important advantage over Lyapunov's direct method. While Lyapunov's direct method usually requires pointwise conditions, fixed point theory needs average conditions; see Burton [1].

In 2001, Burton and Furumochi [2] observed some difficulties that occur in studying the stability theory of ordinary and functional differential equations by Lyapunov's second (direct) method. Rather than inventing new modifications of the standard Lyapunov function(al) method to overcome the difficulties, the authors demonstrate by various examples that the contraction mapping principle can do the magic in many circumstances.

Later, in 2005, by using contraction mappings, Burton [3] investigated the scalar Liénard type equation with constant delay, $L(>0)$ :

$$
\ddot{x}+f(t, x, \dot{x}) \dot{x}+b(t) g(x(t-L))=0 .
$$

Burton [3] obtained conditions for each solution $x(t)$ to satisfy $\left(x(t), x^{\prime}(t)\right) \rightarrow(0,0)$ as $t \rightarrow \infty$.

Later, in 2011, Pi [4] studied stability properties of solutions to a scalar functional Liénard type equation with variable delay, $\tau(t)(>0)$ :

$$
\ddot{x}+f(t, x, \dot{x}) \dot{x}+b(t) g(x(t-\tau(t)))=0 .
$$

By using fixed point theory under an exponentially weighted metric, Pi [4] obtained some interesting sufficient conditions ensuring that the zero solution of this equation is stable and asymptotically stable.

On the other hand, some recent relative results proceeded on the qualitative behaviors of delay differential equations, neutral differential equations, neutral Volterra integrodifferential equations, and certain nonlinear differential equations of second order with and without delay can be summarized as follows.

In [5], Fan et al. studied delay differential equations of the form

$$
\begin{aligned}
& \dot{x}=-a\left(t, x_{t}\right) x(t)+f\left(t, x_{t}\right), \\
& \dot{x}=-g(t, x(t))+f\left(t, x_{t}\right),
\end{aligned}
$$

and the authors established sufficient and necessary criteria for the asymptotic stability by using two different approaches, the contraction mapping principle and Schauder's fixed point theorem. 
Raffoul [6] dealt with the stability of the zero solution of a scalar neutral differential equation. The author established sufficient conditions for the stability of the zero solution on the base of the contraction mapping principle.

In [7], Jin and Luo aimed to study the asymptotic stability for some scalar differential equations of retarded and neutral type by using a fixed point approach. The authors did not use Lyapunov's method; they got interesting results for the stability even when the delay is unbounded. The authors also obtained necessary and sufficient conditions for the asymptotic stability.

Zhang and Liu [8] considered a nonlinear neutral differential equation. By using fixed point theory, they gave some conditions to ensure that the zero solution to the equation is asymptotically stable. Hence, some existing results were improved and generalized by this work.

Ardjouni and Djoudi [9] used the contraction mapping theorem to obtain an asymptotic stability result of the zero solution of a nonlinear neutral Volterra integrodifferential equation with variable delays. The asymptotic stability theorem with a necessary and sufficient condition was proved, which improves and extends the results in the literature.

In 2010, Tunç [10] considered the following Liénard type equation with multiple variable deviating arguments, $\tau_{j}(t)$ :

$$
\begin{gathered}
\ddot{x}(t)+f_{1}(x(t), \dot{x}(t)) \dot{x}(t)+f_{2}(x(t)) \dot{x}(t) \\
+g_{0}(x(t))+\sum_{j=1}^{m} g_{j}\left(x\left(t-\tau_{j}(t)\right)\right) \\
=p\left(t, x(t), x\left(t-\tau_{1}(t)\right), \ldots,\right. \\
\left.x\left(t-\tau_{m}(t)\right), \ldots, \dot{x}\left(t-\tau_{m}(t)\right)\right) .
\end{gathered}
$$

The author studied the problems of stability and boundedness of the solutions of this equation by using the Lyapunov second method and made a comparison with some earlier works in the literature.

In [11], the author considered the nonlinear differential equation of second order with a constant delay, $r$ :

$$
\begin{aligned}
\ddot{x}(t)+\{f(t, x(t), x(t-r), \dot{x}(t), \dot{x}(t-r)) \\
+g(t, x(t), x(t-r), \dot{x}(t), \dot{x}(t-r)) \dot{x}(t)\} \dot{x}(t) \\
+b(t) h(x(t-r))=e(t, x(t), x(t-r), \dot{x}(t), \dot{x}(t-r)) .
\end{aligned}
$$

The author discussed the stability of the zero solution of this equation, when $e(\cdot)=0$, and established two new results on the boundedness and uniform-boundedness of the solutions of the same equation, when $e(\cdot) \neq 0$. By this work, Tunç [11] improved the existing results on the stability and boundedness of the solutions of the differential equations of second order without a delay by imposing a few new criteria to the second order nonlinear and nonautonomous delay differential equations of the above form.
Further, Tunç [12] took into consideration the vector Liénard equation with the multiple constant deviating arguments, $\tau_{i}>0$ :

$$
\begin{gathered}
\ddot{X}(t)+F(X(t), \dot{X}(t)) \dot{X}(t)+G(X(t)) \\
+\sum_{i=1}^{n} H_{i}\left(X\left(t-\tau_{i}\right)\right)=P(t) .
\end{gathered}
$$

Based on the Lyapunov-Krasovskii functional approach, the asymptotic stability of the zero solution and the boundedness of all solutions of this equation, when $P(t)=0$ and $P(t) \neq 0$, respectively, are discussed.

More recently, by using Lyapunov's function and functional approach, Tunç $[13,14]$ and Tunç and Yazgan [15] discussed some problems on stability, the boundedness, and the existence of periodic solutions of a certain second order vector and scalar nonlinear differential equations without and with delay. In [16], Tunç also gave certain sufficient conditions for the existence of periodic solutions to a Rayleigh-type equation with state-dependent delay.

By the mentioned papers, the authors contributed to the subject for a class of ordinary and functional differential equations.

In this paper, instead of the mentioned equations, we consider the scalar Liénard type equation with multiple variable delays:

$$
\ddot{x}+f(t, x, \dot{x}) \dot{x}+\sum_{j=1}^{n} b_{j}(t) g_{j}\left(x\left(t-\tau_{j}(t)\right)\right)=0,
$$

where $t \in \mathfrak{R}^{+}, \mathfrak{R}^{+}=[0, \infty), b_{j}: \mathfrak{R}^{+} \rightarrow \mathfrak{R}^{+}$are bounded and continuous functions, $g_{j}: \mathfrak{R} \rightarrow \mathfrak{R}, g_{j}(0)=0, f: \mathfrak{R}^{+} \times \mathfrak{R} \times$ $\mathfrak{R} \rightarrow \mathfrak{R}^{+}$, and $\tau_{j}: \mathfrak{R}^{+} \rightarrow \mathfrak{R}^{+}$are all continuous functions such that $t-\tau_{j}(t) \geq 0$.

We can write (7) as follows:

$$
\begin{gathered}
\dot{x}=y, \\
\dot{y}=-f(t, x, y) y-\sum_{j=1}^{n} b_{j}(t) g_{j}\left(x\left(t-\tau_{j}(t)\right)\right) .
\end{gathered}
$$

For each $t_{0} \geq 0$, we define $m\left(t_{0}\right)=\inf \left\{s-\tau_{1}(s), \ldots, s-\right.$ $\left.\tau_{n}(s): s \geq t_{0}\right\}$ and $C\left(t_{0}\right)=C\left(\left[m\left(t_{0}\right), t_{0}\right], R\right)$ with the continuous function norm $\|\cdot\|$, where

$$
\|\psi\|=\sup \left\{|\psi(s)|: m\left(t_{0}\right) \leq s \leq t_{0}\right\} .
$$

It will cause no confusion even if we use $\|\phi\|$ as the supremum on $\left[m\left(t_{0}\right), \infty\right)$. It can be seen from [9] that, for a given continuous function $\phi$ and a number $y_{0}$, there exists a solution of system (8) on an interval $\left[t_{0}, T\right)$; if the solution remains bounded, then $T=\infty$. Let $(x(t), y(t))$ denote the solution $\left(x\left(t, \phi, y_{0}\right), y\left(t, \phi, y_{0}\right)\right)$.

Definition 1. The zero solution of system (8) is stable if for each $\varepsilon>0$ there exists $\delta=\delta\left(\varepsilon, t_{0}\right)>0$ such that $[\phi \epsilon$ $\left.C\left(t_{0}\right), y_{0} \in R,\|\phi\|+\left|y_{0}\right|<\delta\right]$ implies that $\left|x\left(t, \phi, y_{0}\right)\right|+$ $\left|y\left(t, \phi, y_{0}\right)\right|<\varepsilon$ for $t \geq t_{0}$. 
We make the following basic assumptions on the delay functions $\tau_{j}(t):\left(A_{1}\right)$. Let $t-\tau_{j}(t)$ be strictly increasing and $\lim _{t \rightarrow \infty}\left(t-\tau_{j}(t)\right)=\infty$. The inverses of $t-\tau_{j}(t)$ exist, denoted by $P_{j}(t)$ and $0 \leq b_{j}(t) \leq M_{j}, j=1,2, \ldots, n$. Let $M=\max \left\{M_{1}, \ldots, M_{n}\right\}$. Hence, $0 \leq b_{j}(t) \leq M$.

It is also worth mentioning that throughout the papers [10-15] the authors discussed the qualitative behavior of solutions of certain scalar and vector ordinary and functional differential equations of second order by means of the Lyapunov function or functional approach. In this paper, instead of the mentioned methods, we use the fixed point technique under an exponentially weighted metric to discuss stability of solutions to a kind of scalar Liénard type equations with multiple variable delays. This approach has a contribution to the topic in the literature, and it may be useful for researchers to work on the qualitative behaviors of solutions.

\section{Main Result}

In this section, sufficient conditions for stability are presented by the fixed point theory. We give some results on stability of the zero solution of (7). Before giving our main result, we introduce some auxiliary results.

Lemma 2. Let $\psi:\left[m\left(t_{0}\right), t_{0}\right] \rightarrow R$ be a given continuous function. If $(x(t), y(t))$ is the solution of system (8) on $\left[t_{0}, T_{1}\right)$ satisfying $(t)=\psi(t), t \in\left[m\left(t_{0}\right), t_{0}\right]$, and $y\left(t_{0}\right)=x^{\prime}\left(t_{0}\right)$, then $x(t)$ is the solution of the following integral equation:

$x(t)$

$$
\begin{aligned}
= & \psi\left(t_{0}\right) e^{-\int_{t_{0}}^{t} K(s) d s}+\int_{t_{0}}^{t} e^{-\int_{u}^{t} K(s) d s} B(u) d u \\
& +\sum_{j=1}^{n} \int_{t_{0}}^{t} e^{-\int_{u}^{t} K(s) d s} \widehat{D}_{j}(u)\left[x(u)-g_{j}(x(u))\right] d u \\
& +\sum_{j=1}^{n} \int_{t-\tau_{j}(t)}^{t} \widehat{D}_{j}(s) g_{j}(x(s)) d s \\
& -\sum_{j=1}^{n} e^{-\int_{t_{0}}^{t} K(s) d s} \int_{t_{0}-\tau_{j}\left(t_{0}\right)}^{t_{0}} \widehat{D}_{j}(s) g_{j}(x(s)) d s \\
& -\sum_{j=1}^{n} \int_{t_{0}}^{t}\left[\int_{u-\tau_{j}(u)}^{u} \widehat{D}_{j}(s) g_{j}(x(s)) d s\right] e^{-\int_{u}^{t} K(s) d s} K(u) d u \\
& +\sum_{j=1}^{n} \int_{t_{0}}^{t} E_{j}(t, s) g_{j}\left(x\left(s-\tau_{j}(s)\right)\right) d s \\
& -\sum_{j=1}^{n} \int_{t_{0}}^{t}\left[\int_{t_{0}}^{u} E_{j}(u, s)\right. \\
\times & \left.\times g_{j}\left(x\left(s-\tau_{j}(s)\right)\right) d s\right] e^{-\int_{u}^{t} K(s) d s} K(u) d u .
\end{aligned}
$$

Conversely, if the continuous function $x(t)=\psi(t), t \in$ $\left[m\left(t_{0}\right), t_{0}\right]$ is the solution of (10) on $\left[t_{0}, T_{2}\right]$, then $(x(t), y(t))$ is the solution of system (8) on $\left[t_{0}, T_{2}\right]$.

Proof. Let $f(t, x(t), y(t))=A(t)$. Then, (8) can be written as the following system:

$$
\begin{gathered}
\dot{x}=y, \\
\dot{y}=-A(t) y-\sum_{j=1}^{n} b_{j}(t) g_{j}\left(x\left(t-\tau_{j}(t)\right)\right)
\end{gathered}
$$

so that

$$
\dot{y}+A(t) y+\sum_{j=1}^{n} b_{j}(t) g_{j}\left(x\left(t-\tau_{j}(t)\right)\right)=0 .
$$

Multiplying both sides of (12) by $e^{\int_{t_{0}}^{t} A(s) d s}$ and then integrating from $t_{0}$ to $t$, we obtain

$$
\begin{aligned}
y(t)= & y\left(t_{0}\right) e^{-\int_{t_{0}}^{t} A(s) d s} \\
& -\int_{t_{0}}^{t} e^{-\int_{u}^{t} A(s) d s} \sum_{j=1}^{n} b_{j}(u) g_{j}\left(x\left(u-\tau_{j}(u)\right)\right) d u,
\end{aligned}
$$

and hence

$$
\begin{aligned}
\dot{x}(t)= & \dot{x}\left(t_{0}\right) e^{-\int_{t_{0}}^{t} A(s) d s} \\
& -\int_{t_{0}}^{t} e^{-\int_{u}^{t} A(s) d s} \sum_{j=1}^{n} b_{j}(u) g_{j}\left(x\left(u-\tau_{j}(u)\right)\right) d u .
\end{aligned}
$$

If we choose $\dot{x}\left(t_{0}\right) e^{-\int_{t_{0}}^{t} A(s) d s}=B(t)$, then it follows that

$$
\dot{x}(t)=B(t)-\sum_{j=1}^{n} \int_{t_{0}}^{t} e^{-\int_{u}^{t} A(s) d s} b_{j}(u) g_{j}\left(x\left(u-\tau_{j}(u)\right)\right) d u .
$$

Let

$$
\begin{gathered}
\sum_{j=1}^{n} e^{-\int_{u}^{t} A(s) d s} b_{j}(u)=\sum_{j=1}^{n} C_{j}(t, u), \\
\sum_{j=1}^{n} \int_{t_{0}}^{\infty} C_{j}\left(u+t-t_{0}, t\right) d u=\sum_{j=1}^{n} D_{j}(t), \\
\sum_{j=1}^{n} \frac{D_{j}(t)}{1-\tau^{\prime}(t)}=\sum_{j=1}^{n} \widetilde{D}_{j}(t), \\
\sum_{j=1}^{n} \widetilde{D}_{j}\left(p_{j}(t)\right)=\sum_{j=1}^{n} \widehat{D}_{j}(t), \\
\sum_{j=1}^{n} \int_{t_{0}+t-s}^{\infty} C_{j}\left(u+s-t_{0}, s\right) d u=\sum_{j=1}^{n} E_{j}(t, s) .
\end{gathered}
$$


Then, (15) can be written in the form of

$$
\begin{aligned}
\dot{x}(t)= & B(t)-\sum_{j=1}^{n} g_{j}\left(x\left(t-\tau_{j}(t)\right)\right) \int_{t_{0}}^{\infty} C_{j}\left(u+t-t_{0}, t\right) d u \\
& +\sum_{j=1}^{n} \frac{d}{d t} \int_{t_{0}}^{t} E_{j}(t, s) g_{j}\left(x\left(s-\tau_{j}(s)\right)\right) d s .
\end{aligned}
$$

Hence

$$
\begin{aligned}
\dot{x}(t)= & B(t)-\sum_{j=1}^{n} g_{j}\left(x\left(t-\tau_{j}(t)\right)\right) D_{j}(t) \\
& +\sum_{j=1}^{n} \frac{d}{d t} \int_{t_{0}}^{t} E_{j}(t, s) g_{j}\left(x\left(s-\tau_{j}(s)\right)\right) d s
\end{aligned}
$$

so that

$$
\begin{aligned}
\dot{x}(t)= & B(t)-\sum_{j=1}^{n} \widetilde{D}_{j}\left(p_{j}(t)\right) g_{j}(x(t)) \\
& +\sum_{j=1}^{n} \frac{d}{d t} \int_{t-\tau_{j}(t)}^{t} \widetilde{D}_{j}\left(p_{j}(s)\right) g_{j}(x(s)) d s \\
& +\sum_{j=1}^{n} \frac{d}{d t} \int_{t_{0}}^{t} E_{j}(t, s) g_{j}\left(x\left(s-\tau_{j}(s)\right)\right) d s .
\end{aligned}
$$

$$
\begin{aligned}
\dot{x}(t)= & B(t)-\sum_{j=1}^{n} \widehat{D}_{j}(t) x(t) \\
& +\sum_{j=1}^{n} \widehat{D}_{j}(t)\left[x(t)-g_{j}(x(t))\right] \\
& +\sum_{j=1}^{n} \frac{d}{d t} \int_{t-\tau_{j}(t)}^{t} \widehat{D}_{j}(s) g_{j}(x(s)) d s \\
& +\sum_{j=1}^{n} \frac{d}{d t} \int_{t_{0}}^{t} E_{j}(t, s) g_{j}\left(x\left(s-\tau_{j}(s)\right)\right) d s .
\end{aligned}
$$

Let $\sum_{j=1}^{n} \widehat{D}_{j}(t)=K(t)$. Then,

$$
\begin{aligned}
\dot{x}(t)+K(t) x(t)= & B(t)+\sum_{j=1}^{n} \widehat{D}_{j}(t)\left[x(t)-g_{j}(x(t))\right] \\
& +\sum_{j=1}^{n} \frac{d}{d t} \int_{t-\tau_{j}(t)}^{t} \widehat{D}_{j}(s) g_{j}(x(s)) d s \\
& +\sum_{j=1}^{n} \frac{d}{d t} \int_{t_{0}}^{t} E_{j}(t, s) g_{j}\left(x\left(s-\tau_{j}(s)\right)\right) d s .
\end{aligned}
$$

Multiplying both sides of (21) by $e^{\int_{t_{0}}^{t} K(s) d s}$ and then integrating from $t_{0}$ to $t$, then

$$
\begin{aligned}
\int_{t_{0}}^{t}\left[x(u) e^{\int_{t_{0}}^{t} K(s) d s}\right]^{\prime} d u \\
=\int_{t_{0}}^{t} e^{\int_{t_{0}}^{u} K(s) d s} B(u) d u \\
\quad+\sum_{j=1}^{n} \int_{t_{0}}^{t} e^{\int_{t_{0}}^{u} K(s) d s} \widehat{D}_{j}(u)\left[x(u)-g_{j}(x(u))\right] d u \\
\quad+\sum_{j=1}^{n} \int_{t_{0}}^{t} e^{\int_{t_{0}}^{u} K(s) d s} \frac{d}{d t} \int_{t-\tau_{j}(t)}^{t} \widehat{D}_{j}(s) g_{j}(x(s)) d s d u \\
\quad+\sum_{j=1}^{n} \int_{t_{0}}^{t} e^{\int_{t_{0}}^{u} K(s) d s} \frac{d}{d t} \int_{t_{0}}^{t} E_{j}(t, s) g_{j}\left(x\left(s-\tau_{j}(s)\right)\right) d s d u
\end{aligned}
$$

so that

$$
\begin{aligned}
& x(t) \\
& =\psi\left(t_{0}\right) e^{-\int_{t_{0}}^{t} K(s) d s}+\int_{t_{0}}^{t} e^{-\int_{u}^{t} K(s) d s} B(u) d u \\
& +\sum_{j=1}^{n} \int_{t_{0}}^{t} e^{-\int_{u}^{t} K(s) d s} \widehat{D}_{j}(u)\left[x(u)-g_{j}(x(u))\right] d u \\
& +\sum_{j=1}^{n} \int_{t_{0}}^{t} e^{-\int_{u}^{t} K(s) d s}\left[\frac{d}{d u} \int_{u-\tau_{j}(u)}^{u} \widehat{D}_{j}(s) g_{j}(x(s)) d s\right] d u \\
& +\sum_{j=1}^{n} \int_{t_{0}}^{t} e^{-\int_{u}^{t} K(s) d s} \\
& \quad \times\left[\frac{d}{d u} \int_{t_{0}}^{u} E_{j}(u, s) g_{j}\left(x\left(s-\tau_{j}(s)\right)\right) d s\right] d u .
\end{aligned}
$$

Applying the integration by parts formula for the last two terms, we have

$x(t)$

$$
\begin{aligned}
= & \psi\left(t_{0}\right) e^{-\int_{t_{0}}^{t} K(s) d s}+\int_{t_{0}}^{t} e^{-\int_{u}^{t} K(s) d s} B(u) d u \\
& +\sum_{j=1}^{n} \int_{t_{0}}^{t} e^{-\int_{u}^{t} K(s) d s} \widehat{D}_{j}(u)\left[x(u)-g_{j}(x(u))\right] d u \\
& +\sum_{j=1}^{n} \int_{t-\tau_{j}(t)}^{t} \widehat{D}_{j}(s) g_{j}(x(s)) d s \\
& -\sum_{j=1}^{n} e^{-\int_{t_{0}}^{t} K(s) d s \int_{t_{0}-\tau_{j}\left(t_{0}\right)}^{t_{0}} \widehat{D}_{j}(s) g_{j}(x(s)) d s}
\end{aligned}
$$




$$
\begin{aligned}
& -\sum_{j=1}^{n} \int_{t_{0}}^{t}\left[\int_{u-\tau_{j}(u)}^{u} \widehat{D}_{j}(s) g_{j}(x(s)) d s\right] e^{-\int_{u}^{t} K(s) d s} K(u) d u \\
& +\sum_{j=1}^{n} \int_{t_{0}}^{t} E_{j}(t, s) g_{j}\left(x\left(s-\tau_{j}(s)\right)\right) d s \\
& -\sum_{j=1}^{n} \int_{t_{0}}^{t}\left[\int_{t_{0}}^{u} E_{j}(u, s)\right. \\
& \left.\quad \times g_{j}\left(x\left(s-\tau_{j}(s)\right)\right) d s\right] e^{-\int_{u}^{t} K(s) d s} K(u) d u .
\end{aligned}
$$

Conversely, we assume that a continuous function $x(t)=$ $\psi(t)$ for $t \in\left[m\left(t_{0}\right), t_{0}\right]$ and satisfies the integral equation on $t \in\left[t_{0}, T_{2}\right]$. Then, it is differentiable on $\left[t_{0}, T_{2}\right]$. Hence, it is only needed to differentiate the integral equation. When we differentiate the integral equation, we can conclude the desired result.

Let $(C,\|\cdot\|)$ be the Banach space of bounded continuous functions on $\left[m\left(t_{0}\right), \infty\right)$ with the supremum norm $\|\phi\|=$ $\sup \left\{|\phi(t)|: t \in\left[m\left(t_{0}\right), \infty\right)\right\}$ for $\phi \in C$. Let $\rho$ denote the supremum metric and $\rho\left(\phi_{1}, \phi_{2}\right)=\left\|\phi_{1}-\phi_{2}\right\|$, where $\phi_{1}, \phi_{2} \in C$. Next, let $\psi:\left[m\left(t_{0}\right), t_{0}\right] \rightarrow R$ be a given continuous initial function.

Define the set $S \subset C$ by

$$
\begin{array}{r}
S=\left\{\phi:\left[m\left(t_{0}\right), \infty\right) \longrightarrow R \mid \phi \in C,\right. \\
\left.\phi(t)=\psi(t), t \in\left[m\left(t_{0}\right), t_{0}\right]\right\}
\end{array}
$$

and its subset

$$
\begin{aligned}
S^{\prime}=\left\{\phi:\left[m\left(t_{0}\right), \infty\right) \longrightarrow R \mid \phi \in C, \phi(t)\right. \\
\left.=\psi(t), t \in\left[m\left(t_{0}\right), t_{0}\right],|\phi(t)| \leq l, t \geq m\left(t_{0}\right)\right\},
\end{aligned}
$$

where $\psi:\left[m\left(t_{0}\right), t_{0}\right] \rightarrow[-l, l]$ is a given initial function and $l$ is a positive constant. Define the mapping $P: S^{\prime} \rightarrow S^{\prime}$ by

$$
(P \phi)(t)=\psi(t), \quad \text { if } t \in\left[m\left(t_{0}\right), t_{0}\right]
$$

and if $t>t_{0}$, then

$$
\begin{aligned}
(P \phi)(t)= & \psi\left(t_{0}\right) e^{-\int_{t_{0}}^{t} K(s) d s} \\
& +\int_{t_{0}}^{t} e^{-\int_{u}^{t} K(s) d s} B(u) d u \\
& +\sum_{j=1}^{n} \int_{t_{0}}^{t} e^{-\int_{u}^{t} K(s) d s} \widehat{D}_{j}(u)\left[\phi(u)-g_{j}(\phi(u))\right] d u
\end{aligned}
$$

$$
\begin{aligned}
& +\sum_{j=1}^{n} \int_{t-\tau_{j}(t)}^{t} \widehat{D}_{j}(s) g_{j}(\phi(s)) d s \\
& -\sum_{j=1}^{n} e^{-\int_{t_{0}}^{t} K(s) d s} \int_{t_{0}-\tau_{j}\left(t_{0}\right)}^{t_{0}} \widehat{D}_{j}(s) g_{j}(\psi(s)) d s \\
& +\sum_{j=1}^{n} \int_{t_{0}}^{t} E_{j}(t, s) g_{j}(\phi(s)) d s \\
& -\sum_{j=1}^{n} \int_{t_{0}}^{t}\left[\int_{u-\tau_{j}(u)}^{u} \widehat{D}_{j}(s)\right. \\
& \left.\times g_{j}(\phi(s)) d s\right] e^{-\int_{u}^{t} K(s) d s} K(u) d u \\
& -\sum_{j=1}^{n} \int_{t_{0}}^{t}\left[\int_{t_{0}}^{u} E_{j}(u, s)\right. \\
& \left.\times g_{j}\left(\phi\left(s-\tau_{j}(s)\right)\right) d s\right] e^{-\int_{u}^{t} K(s) d s} \\
& \times K(u) d u \text {. }
\end{aligned}
$$

Since $g_{j}(x)$ satisfy the Lipschitz condition, let $L_{1}, \ldots, L_{n}$ denote the common Lipschitz constants for $g_{j}(x)$ and $x-$ $g_{j}(x)$.

It is also clear that

$$
\begin{aligned}
\int_{t_{0}}^{t} e^{-\int_{u}^{t} K(s) d s} K(u) d u & =\left.e^{-\int_{u}^{t} K(s) d s}\right|_{t_{0}} ^{t} \\
& =1-e^{-\int_{t_{0}}^{t} K(s) d s} \approx 1, \quad \text { for large } t .
\end{aligned}
$$

But since $g_{j}(x)$ are nonlinear, then $L_{j}$ may not be small enough. Hence, $P$ may not be a contracting mapping. We can solve this problem by giving an exponentially weight metric via the next lemma.

Lemma 3. We suppose that there exists a constant $l>0$ such that $g_{j}(x)$ satisfy the Lipschitz condition on $[-l, l]$. Then there exists a metric on $S^{\prime}$ such that

(i) the metric space $\left(S^{\prime}, d\right)$ is complete,

(ii) $P$ is a contraction mapping on $\left(S^{\prime}, d\right)$ if $P$ maps $S^{\prime}$ into itself.

Proof. (i) We change the supremum norm to an exponentially weighted norm $|\phi|_{h}$, which is defined on $S^{\prime}$. Let $X$ be the space of all continuous functions $\phi:\left[m\left(t_{0}\right), \infty\right) \rightarrow R$ such that

$$
|\phi|_{h}=\sup _{n}\left\{|\phi(t)| e^{-h(t)}: t \in\left[m\left(t_{0}\right), \infty\right)\right\}<\infty
$$

where $h(t)=k \sum_{j=1}^{n} L_{j} \int_{t_{0}}^{t}\left[\widehat{D}_{j}(s)+D_{j}(s)\right] d s, k$ is a constant, and $L_{j}$ are the common Lipschitz constants for $x-g_{j}(x)$ and $g_{j}(x)$. Then $\left(X,|\cdot|_{h}\right)$ is a Banach space. Thus $(X, d)$ is a 
complete metric space with $d(\phi, \varphi)=|\phi-\varphi|_{h}$, where $\phi, \varphi \in$ $S$. Under this metric, the space $S^{\prime}$ is a closed subset of $X$. Thus the metric space $\left(S^{\prime}, d\right)$ is complete.

(ii) Let $P: S^{\prime} \rightarrow S^{\prime}$. It is clear that $\sum_{j=1}^{n} \widehat{D}_{j}(t) \geq 0$ and $\sum_{j=1}^{n} E_{j}(t, s) \geq 0$. Then, for $\phi, \varphi \in S^{\prime}$, we can get

$$
\begin{aligned}
& |(P \phi)(t)-(P \varphi)(t)| e^{-h(t)} \\
& \leq \sum_{j=1}^{n} \int_{t_{0}}^{t} e^{-\int_{u}^{t} K(s) d s} \widehat{D}_{j}(u) \mid\left[\phi(u)-g_{j}(\phi(u))\right] \\
& -\left[\varphi(u)-g_{j}(\varphi(u))\right] \mid e^{-h(t)} d u \\
& +\sum_{j=1}^{n} \int_{t_{0}}^{t} E_{j}(t, s)\left|g_{j}(\phi(s))-g_{j}(\varphi(s))\right| e^{-h(t)} d s \\
& +\sum_{j=1}^{n} \int_{t-\tau_{j}(t)}^{t} \widetilde{D}_{j}(s)\left|g_{j}(\phi(s))-g_{j}(\varphi(s))\right| e^{-h(t)} d s \\
& +\sum_{j=1}^{n} \int_{t_{0}}^{t}\left[\int_{u-\tau_{j}(u)}^{u} \widetilde{D}_{j}(s) d s \mid g_{j}(\phi(s))\right. \\
& \left.-g_{j}(\varphi(s)) \mid e^{-h(t)} d s\right] \\
& \times e^{-\int_{u}^{t} K(s) d s} K(u) d u \\
& +\sum_{j=1}^{n} \int_{t_{0}}^{t}\left[\int_{t_{0}}^{u} E_{j}(u, s) \mid g_{j}\left(\phi\left(s-\tau_{j}(s)\right)\right)\right. \\
& \left.-g_{j}\left(\varphi\left(s-\tau_{j}(s)\right)\right) \mid e^{-h(t)} d s\right] \\
& \times e^{-\int_{u}^{t} K(s) d s} K(u) d u \text {. }
\end{aligned}
$$

For $u \leq t$, since $D_{j}(t) \geq 0$, we have

$$
\begin{aligned}
h(u)-h(t)= & \sum_{j=1}^{n} k L_{j} \int_{t_{0}}^{u}\left[\widehat{D}_{j}(s)+D_{j}(s)\right] d s \\
& -\sum_{j=1}^{n} k L_{j} \int_{t_{0}}^{t}\left[\widehat{D}_{j}(s)+D_{j}(s)\right] d s \\
= & \sum_{j=1}^{n}(-k) L_{j} \int_{u}^{t}\left[\widehat{D}_{j}(s)+D_{j}(s)\right] d s \\
\leq & \sum_{j=1}^{n}(-k) L_{j} \int_{u}^{t} \widehat{D}_{j}(s) d s .
\end{aligned}
$$

Further for $s \leq t$, it follows that

$$
\begin{aligned}
h(s & \left.-\tau_{j}(s)\right)-h(t) \\
= & \sum_{j=1}^{n} k L_{j} \int_{t_{0}}^{s-\tau_{j}(s)}\left[\widehat{D}_{j}(s)+D_{j}(s)\right] d s \\
& -\sum_{j=1}^{n} k L_{j} \int_{t_{0}}^{t}\left[\widehat{D}_{j}(s)+D_{j}(s)\right] d s \\
= & \sum_{j=1}^{n}(-k) L_{j} \int_{s-\tau_{j}(s)}^{t}\left[\widehat{D}_{j}(u)+D_{j}(u)\right] d u \\
\leq & \sum_{j=1}^{n}(-k) L_{j} \int_{s}^{t} D_{j}(u) d u .
\end{aligned}
$$

Since $E(t, s) \geq 0$, then we have

$$
\begin{aligned}
\sum_{j=1}^{n} E_{j}(t, s) & =\sum_{j=1}^{n} \int_{t_{0}+t-s}^{\infty} c_{j}\left(u+s-t_{0}, s\right) d u \\
& \leq \sum_{j=1}^{n} \int_{t_{0}}^{\infty} c_{j}\left(u+s-t_{0}, s\right) d u=\sum_{j=1}^{n} D_{j}(s) .
\end{aligned}
$$

Hence

$$
\begin{aligned}
& \mid(P \phi)(t)-(P \varphi)(t) \mid e^{-h(t)} \\
& \leq \mid \phi-\left.\varphi\right|_{h} \\
& \times\left\{\sum_{j=1}^{n} L_{j} \int_{t_{0}}^{t} e^{-\int_{u}^{t} K(s) d s} \widehat{D}_{j}(u) e^{h(u)-h(t)} d u\right. \\
&+\sum_{j=1}^{n} L_{j} \int_{t_{0}}^{t} E_{j}(t, s) e^{h(s)-h(t)} d s \\
&+\sum_{j=1}^{n} L_{j} \int_{t-\tau_{j}(t)}^{t} \widehat{D}_{j}(s) e^{h(s)-h(t)} d s \\
&+\sum_{j=1}^{n} L_{j} \int_{t_{0}}^{t}\left[\int_{u-\tau_{j}(t)}^{u} \widehat{D}_{j}(s) e^{h(s)-h(t)} d s\right] \\
& \times e^{-\int_{u}^{t} K(s) d s} K(u) d u \\
&+\sum_{j=1}^{n} L_{j} \int_{t_{0}}^{t}\left[\int_{t_{0}}^{u} E_{j}(u, s) e^{h\left(s-\tau_{j}(s)\right)-h(t)} d s\right] \\
&\left.\times e^{-\int_{u}^{t} K(s) d s} K(u) d u\right\} .
\end{aligned}
$$


Therefore,

$$
\begin{aligned}
& \sum_{j=1}^{n} L_{j} \int_{t_{0}}^{t} e^{-\int_{u}^{t} K(s) d s} \widehat{D}_{j}(u) e^{h(u)-h(t)} d u \\
& \quad=\sum_{j=1}^{n} L_{j} \int_{t_{0}}^{t} e^{-\sum_{j=1}^{n} \int_{u}^{t} \widehat{D}_{j}(s) d s} \widehat{D}_{j}(u) e^{h(u)-h(t)} d u \\
& \quad \leq \sum_{j=1}^{n} L_{j} \int_{t_{0}}^{t} \frac{e^{-\sum_{j=1}^{n} \int_{u}^{t} \widehat{D}_{j}(s) d s} \widehat{D}_{j}(u)}{e^{\sum_{j=1}^{n} k L_{j} \int_{u}^{t} \widehat{D}_{j}(s) d s} d u} \\
& \quad=\sum_{j=1}^{n} L_{j} \int_{t_{0}}^{t} e^{-\sum_{j=1}^{n}\left(k L_{j}+1\right) \int_{u}^{t} \widehat{D}_{j}(s) d s} \widehat{D}_{j}(u) d u \\
& \quad \leq\left.\sum_{j=1}^{n} L_{j} \frac{1}{\sum_{j=1}^{n}\left(k L_{j}+1\right)} e^{-\sum_{j=1}^{n}\left(k L_{j}+1\right) \int_{u}^{t} \widehat{D}_{j}(s) d s}\right|_{t_{0}} ^{t} \\
& \quad \leq \sum_{j=1}^{n} L_{j} \frac{1}{\sum_{j=1}^{n} k L_{j}} \leq \frac{1}{k}, \\
& \sum_{j=1}^{n} L_{j} \int_{t_{0}}^{t} E_{j}(t, s) e^{h(s)-h(t)} d s \\
& \quad \leq \sum_{j=1}^{n} L_{j} \int_{t_{0}}^{t} D_{j}(s) e^{\sum_{j=1}^{n}(-k) L_{j} \int_{s}^{t} D_{j}(s) d s} d s \\
& \quad \leq\left.\sum_{j=1}^{n} L_{j} \frac{1}{\sum_{j=1}^{n} k L_{j}} e^{-\sum_{j=1}^{n} k L_{j} \int_{u}^{t} D_{j}(s) d s}\right|_{t_{0}} ^{t} \leq \frac{1}{k} .
\end{aligned}
$$

Thus, we have

$$
|(P \phi)(t)-(P \varphi)(t)| e^{-h(t)} \leq \frac{5}{k}|\phi-\varphi|_{h}, \quad t>t_{0} .
$$

For $t \in\left[m\left(t_{0}\right), t_{0}\right],(P \phi)(t)=(P \varphi)(t)=\theta(t)$. Thus,

$$
d(P \phi, P \varphi) \leq \frac{5}{k} d(\phi-\varphi), \quad(k>5) .
$$

Therefore, $P$ is contraction mapping on $\left(S^{\prime}, d\right)$.

Theorem 4. We suppose that the assumption $\left(A_{1}\right)$ holds. Moreover, we assume the following.

(i) There exists a positive constant $l$ such that $g_{j}$ satisfy the Lipschitz condition on $[-l, l]$ and $g_{j}$ are odd and they are strictly increasing on $[-l, l]$, and $x-g_{j}(x)$ are nondecreasing on $[-l, l]$.

(ii) There exist an $\alpha_{j} \in(0,1)$ and a continuous function $a(t):[0, \infty) \rightarrow[0, \infty)$ such that $f(t, x, y) \geq a(t)$ for $t \geq 0, x \in R, y \in R$,

$$
\begin{aligned}
& 2 \sup _{t \geq 0} \int_{t}^{P_{j}(t)} \int_{0}^{\infty} e^{-\int_{s}^{w+s} a(v) d v} b_{j}(s) d w d s \\
& \quad+2 \sup _{t \geq 0} \int_{0}^{t} \int_{t-s}^{\infty} e^{-\int_{s}^{w+s} a(v) d v} b_{j}(s) d w d s \leq \alpha_{j} .
\end{aligned}
$$

(iii) There exist constants $a_{0}>0$ and $Q>0$ such that, for each $t \geq 0$, if $J \geq Q$, then

$$
\int_{t}^{t+J} a(v) d v \geq a_{0} J
$$

Then there exists $\delta \in(0, l)$ such that, for each initial function $\psi:\left[m\left(t_{0}\right), t_{0}\right] \rightarrow R$ and $\dot{x}\left(t_{0}\right)$ satisfying $\left|\dot{x}\left(t_{0}\right)\right|+\|\psi\| \leq \delta$, there is a unique continuous function $x:\left[m\left(t_{0}\right), \infty\right) \rightarrow R$ satisfying $x(t)=\psi(t)$, which is a solution of $(7)$ on $\left[t_{0}, \infty\right)$. Moreover, the zero solution of (7) is stable.

Proof. Choose $\psi:\left[m\left(t_{0}\right), t_{0}\right] \rightarrow R$ and $\dot{x}\left(t_{0}\right)$ such that

$$
\begin{aligned}
& \left(Q+\frac{e^{-a_{0} \cdot Q}}{a_{0}}\right)\left|\dot{x}\left(t_{0}\right)\right|+\delta+\sum_{j=1}^{n} g_{j}(\delta) \int_{t_{0}-\tau_{j}\left(t_{0}\right)}^{t_{0}} \widehat{D}_{j}(s) d s \\
& \leq\left[1-\left(\alpha_{1}+\alpha_{2}+\cdots+\alpha_{n}\right)\right] \sum_{j=1}^{n} g_{j}(l) .
\end{aligned}
$$

In view of the assumptions and $g_{j}(0)=0$, it follows that $g_{j}(l) \leq l$. Since $g_{j}(x)$ satisfies Lipschitz condition on $[-l, l]$, thus $g_{j}(x)$ is continuous function on $[-l, l]$. Then, there exists a constant $\delta$ such that $\delta<l$.

Thus, we can get

$$
\begin{aligned}
\mid(P \phi) & (t) \mid \\
\leq & \delta+\int_{t_{0}}^{t} e^{-\int_{u}^{t} K(s) d s}\left|\dot{x}\left(t_{0}\right)\right| e^{-\int_{t_{0}}^{u} A(s) d s} d u \\
& +\sum_{j=1}^{n} \int_{t_{0}}^{t} e^{-\int_{u}^{t} K(s) d s} \widehat{D}_{j}(u)\left(l-g_{j}(l)\right) d u \\
& +\sum_{j=1}^{n} \int_{t-\tau_{j}(t)}^{t} \widehat{D}_{j}(s) g_{j}(l) d s \\
& +\sum_{j=1}^{n} \int_{t_{0}}^{t}\left[\int_{u-\tau_{j}(u)}^{u} \widehat{D}_{j}(s) g_{j}(l) d s\right] e^{-\int_{u}^{t} K(s) d s} K(u) d u \\
& +\sum_{j=1}^{n} \int_{t_{0}-\tau_{j}\left(t_{0}\right)}^{t_{0}} \widehat{D}_{j}(s) g_{j}(\delta) d s \\
& +\sum_{j=1}^{n} \int_{t_{0}}^{t} E_{j}(t, s) g_{j}(l) d s \\
& +\sum_{j=1}^{n} \int_{t_{0}}^{t}\left[\int_{t_{0}}^{u} E_{j}(u, s) g_{j}(l) d s\right] e^{-\int_{u}^{t} K(s) d s} K(u) d u .
\end{aligned}
$$


It also follows that

$$
\begin{aligned}
& \sum_{j=1}^{n} \int_{t_{0}}^{t} E_{j}(t, s) d s=\sum_{j=1}^{n} \int_{t_{0}}^{t} \int_{t_{0}+t-s}^{\infty} C_{j}\left(u+s-t_{0}, s\right) d u d s \\
& =\sum_{j=1}^{n} \int_{t_{0}}^{t} \int_{t_{0}+t-s}^{\infty} e^{-\int_{s}^{u+s-t_{0}} A(v) d v} b_{j}(s) d u d s \\
& =\sum_{j=1}^{n} \int_{t_{0}}^{t} \int_{t-s}^{\infty} e^{-\int_{s}^{u+s} A(v) d v} b_{j}(s) d u d s \\
& \leq \sup _{t \geq 0} \int_{0}^{t} \int_{t-s}^{\infty} e^{-\int_{s}^{u+s} a(v) d v} b_{1}(s) d u d s \\
& +\cdots+\sup _{t \geq 0} \int_{0}^{t} \int_{t-s}^{\infty} e^{-\int_{s}^{u+s} a(v) d v} b_{n}(s) d u d s, \\
& \sum_{j=1}^{n} \int_{t-\tau_{j}(t)}^{t} \widehat{D}_{j}(s) d s=\sum_{j=1}^{n} \int_{t-\tau_{j}(t)}^{t} \widetilde{D}_{j}\left(P_{j}(s)\right) d s \\
& =\sum_{j=1}^{n} \int_{t-\tau_{j}(t)}^{t} \frac{D_{j}\left(P_{j}(s)\right)}{1-\tau_{j}^{\prime}(s)} d s=\sum_{j=1}^{n} \int_{t}^{P_{j}(t)} D_{j}(s) d s \\
& =\sum_{j=1}^{n} \int_{t}^{P_{j}(t)} \int_{0}^{\infty} e^{-\int_{s}^{w+s} a(v) d v} \cdot b_{j}(s) d w d s \\
& \leq \sup _{t \geq 0} \int_{t}^{P_{1}(t)} \int_{0}^{\infty} e^{-\int_{s}^{w+s} a(v) d v} b_{1}(s) d w d s \\
& +\cdots+\sup _{t \geq 0} \int_{t}^{P_{n}(t)} \int_{0}^{\infty} e^{-\int_{s}^{w+s} a(v) d v} b_{n}(s) d w d s .
\end{aligned}
$$

From assumption (ii), we have

$$
\begin{aligned}
& \sum_{j=1}^{n} \int_{t_{0}}^{t} E_{j}(t, s) g_{j}(l) d s \\
& \quad+\sum_{j=1}^{n} \int_{t_{0}}^{t}\left[\int_{u-\tau_{j}(u)}^{u} \widehat{D}_{j}(s) g_{j}(l) d s\right] e^{-\int_{u}^{t} K(s) d s} K(u) d u \\
& +\sum_{j=1}^{n} \int_{t-\tau_{j}(t)}^{t} \widehat{D}_{j}(s) g_{j}(l) d s \\
& \quad+\sum_{j=1}^{n} \int_{t_{0}}^{t}\left[\int_{t_{0}}^{u} E_{j}(u, s) g_{j}(l) d s\right] e^{-\int_{u}^{t} K(s) d s} K(u) d u \\
& \leq \sum_{j=1}^{n} g_{j}(l)\left\{2 \sup _{t \geq 0}^{t} \int_{0}^{\infty} \int_{t-s}^{-\int_{s}^{u+s} a(v) d v} b_{1}(s) d u d s\right. \\
& \quad+\cdots+2 \sup _{t \geq 0} \int_{0}^{t} \int_{t-s}^{\infty} e^{-\int_{s}^{u+s} a(v) d v} b_{n}(s) d u d s
\end{aligned}
$$

$$
\begin{gathered}
+2 \sup _{t \geq 0} \int_{t}^{P_{1}(t)} \int_{0}^{\infty} e^{-\int_{s}^{w+s} a(v) d v} b_{1}(s) d w d s \\
\left.+\cdots+2 \sup _{t \geq 0} \int_{t}^{P_{n}(t)} \int_{0}^{\infty} e^{-\int_{s}^{w+s} a(v) d v} b_{n}(s) d w d s\right\} \\
\leq\left(\alpha_{1}+\alpha_{2}+\cdots+\alpha_{n}\right) \sum_{j=1}^{n} g_{j}(l) .
\end{gathered}
$$

Hence

$$
\begin{aligned}
|(P \phi)(t)| \leq & \delta+\sum_{j=1}^{n} g_{j}(\delta) \int_{t_{0}-\tau_{j}\left(t_{0}\right)}^{t_{0}} \widehat{D}_{j}(s) d s+\sum_{j=1}^{n}\left(l-g_{j}(l)\right) \\
& +\left(\alpha_{1}+\alpha_{2}+\cdots+\alpha_{n}\right) \sum_{j=1}^{n} g_{j}(l) \\
& +\int_{t_{0}}^{t} e^{-\int_{u}^{t} K(s) d s}\left|\dot{x}\left(t_{0}\right)\right| e^{-\int_{t_{0}}^{u} A(s) d s} d u \\
\leq & \delta+\sum_{j=1}^{n} g_{j}(\delta) \int_{t_{0}-\tau_{j}\left(t_{0}\right)}^{t_{0}} \widehat{D}_{j}(s) d s+\sum_{j=1}^{n}\left(l-g_{j}(l)\right) \\
& +\left(\alpha_{1}+\alpha_{2}+\cdots+\alpha_{n}\right) \sum_{j=1}^{n} g_{j}(l) \\
& +\int_{t_{0}}^{t}\left|\dot{x}\left(t_{0}\right)\right| e^{-\int_{t_{0}}^{u} A(s) d s} d u .
\end{aligned}
$$

Using condition (iii) of the theorem, we get

$$
\begin{aligned}
\int_{t_{0}}^{t} e^{-\int_{t_{0}}^{u} A(s) d s} d u & =\int_{t_{0}}^{t_{0}+Q} e^{-\int_{t_{0}}^{u} A(s) d s} d u+\int_{t_{0}+Q}^{t} e^{-\int_{t_{0}}^{u} A(s) d s} d u \\
& \leq Q+\frac{e^{-a_{0} \cdot Q}}{a_{0}} .
\end{aligned}
$$

Thus,

$$
\begin{aligned}
& |(P \phi)(t)| \\
& \leq \delta+\sum_{j=1}^{n} g_{j}(\delta) \int_{t_{0}-\tau_{j}\left(t_{0}\right)}^{t_{0}} \widetilde{D}_{j}(s) d s+\sum_{j=1}^{n}\left(l-g_{j}(l)\right) \\
& \quad+\left(\alpha_{1}+\alpha_{2}+\cdots+\alpha_{n}\right) \sum_{j=1}^{n} g_{j}(l)+\left|\dot{x}\left(t_{0}\right)\right|\left(Q+\frac{e^{-a_{0} \cdot Q}}{a_{0}}\right)
\end{aligned}
$$

and so

$$
\left|\left(P_{2} \phi\right)(t)\right| \leq \sum_{j=1}^{n} l .
$$

It is obvious that if $t \in\left[m\left(t_{0}\right), t_{0}\right]$, then $\left(P_{2} \phi\right)(t)=\psi(t)$. Moreover, for $t \in\left[m\left(t_{0}\right), \infty\right)$, we get $\left|\left(P_{2} \phi\right)(t)\right| \leq \sum_{j=1}^{n} l$. 
Therefore, $P \phi: S^{\prime} \rightarrow S^{\prime}$. Since $P$ is a contraction mapping, then $P$ has unique fixed point $x(t)$ such that $|x(t)| \leq \sum_{j=1}^{n} l$.

From (14), we have

$$
\begin{aligned}
|y(t)| \leq & \left|\dot{x}\left(t_{0}\right)\right| \\
& +\sum_{j=1}^{n} \int_{t_{0}}^{t} e^{-\int_{u}^{t} A(s) d s} b_{j}(u)\left|g_{j}\left(x\left(u-\tau_{j}(u)\right)\right)\right| d u .
\end{aligned}
$$

Since, for $t \in[0, \infty), 0 \leq b_{j}(t) \leq M_{j}$, then

$$
\begin{aligned}
|y(t)| & \leq\left|\dot{x}\left(t_{0}\right)\right|+\sum_{j=1}^{n} M_{j} \int_{t_{0}}^{t} e^{-\int_{u}^{t} A(s) d s}\left|x\left(u-\tau_{j}(u)\right)\right| d u \\
& \leq \sum_{j=1}^{n} l\left(1+M_{j} \int_{t_{0}}^{t} e^{-\int_{u}^{t} A(s) d s} d u\right) \\
& <\sum_{j=1}^{n} l\left[1+M_{j}\left(Q+\frac{e^{-a_{0} Q}}{a_{0}}\right)\right] .
\end{aligned}
$$

Hence

$$
|x(t)|+|y(t)|<\sum_{j=1}^{n} l\left[2+M_{j}\left(Q+\frac{e^{-a_{0} Q}}{a_{0}}\right)\right] .
$$

If we replace $\varepsilon$ by $l$, then we show that the zero solution of (7) is stable. This result completes the proof of the theorem.

\section{Conclusion}

A kind of scalar Liénard type equations with multiple variable delays is considered. The stability of the zero solution of this equation is investigated. In proving our main result, we use the fixed points theory by giving an exponentially weight metric. Our result extends and improves some recent results in the literature.

\section{Conflict of Interests}

The authors declare that there is no conflict of interests regarding the publication of this paper.

\section{Acknowledgment}

The authors of this paper would like to expresses their sincere appreciation to the anonymous referees for their valuable comments and suggestions which have led to an improvement in the presentation of the paper.

\section{References}

[1] T. A. Burton, Stability by Fixed Point Theory for Functional Differential Equations, Dover Publications, Mineola, NY, USA, 2006.
[2] T. A. Burton and T. Furumochi, "Fixed points and problems in stability theory for ordinary and functional differential equations," Dynamic Systems and Applications, vol. 10, no. 1, pp. 89-116, 2001.

[3] T. A. Burton, "Fixed points, stability, and exact linearization," Nonlinear Analysis: Theory, Methods \& Applications, vol. 61, no. 5, pp. 857-870, 2005.

[4] D. Pi, "Study the stability of solutions of functional differential equations via fixed points," Nonlinear Analysis: Theory, Methods \& Applications, vol. 74, no. 2, pp. 639-651, 2011.

[5] M. Fan, Z. Xia, and H. Zhu, "Asymptotic stability of delay differential equations via fixed point theory and applications," Canadian Applied Mathematics Quarterly, vol. 18, no. 4, pp. 361380, 2010.

[6] Y. N. Raffoul, "Stability in neutral nonlinear differential equations with functional delays using fixed-point theory," Mathematical and Computer Modelling, vol. 40, no. 7-8, pp. 691-700, 2004.

[7] C. Jin and J. Luo, "Fixed points and stability in neutral differential equations with variable delays," Proceedings of the American Mathematical Society, vol. 136, no. 3, pp. 909-918, 2008.

[8] R. Zhang and G. Liu, "Stability of nonlinear neutral differential equation via fixed point," Annals of Differential Equations, vol. 28, no. 4, pp. 488-493, 2012.

[9] A. Ardjouni and A. Djoudi, "Fixed points and stability in nonlinear neutral Volterra integro-differential equations with variable delays," Electronic Journal of Qualitative Theory of Differential Equations, no. 20-37, pp. 1-13, 2013.

[10] C. Tunç, "New stability and boundedness results of Liénard type equations with multiple deviating arguments," Journal of Contemporary Mathematical Analysis, vol. 45, no. 4, pp. 47-56, 2010.

[11] C. Tunç, "Stability, boundedness and uniform boundedness of solutions of nonlinear delay differential equations," Discrete and Continuous Dynamical Systems A, pp. 1395-1403, 2011.

[12] C. Tunç, "Stability and boundedness in multi delay Lienard equation," Filomat, vol. 27, no. 3, pp. 437-447, 2013.

[13] C. Tunç, "Stability to vector Liénard equation with constant deviating argument," Nonlinear Dynamics, vol. 73, no. 3, pp. 1245-1251, 2013.

[14] C. Tunç, "A note on the bounded solutions to $x^{\prime \prime}+c\left(t, x, x^{\prime}\right)+$ $q(t) b(x)=f(t)$," Applied Mathematics \& Information Sciences, vol. 8, no. 1, pp. 393-399, 2014.

[15] C. Tunç and R. Yazgan, "Existence of periodic solutions to multidelay functional differential equations of second order," Abstract and Applied Analysis, vol. 2013, Article ID 968541, 5 pages, 2013.

[16] C. Tunç, "New results on the existence of periodic solutions for Rayleigh equation with state-dependent delay," Journal of Mathematical \& Fundamental Sciences, vol. 45, no. 2, pp. 154162,2013 


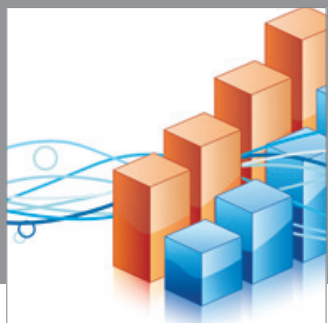

Advances in

Operations Research

mansans

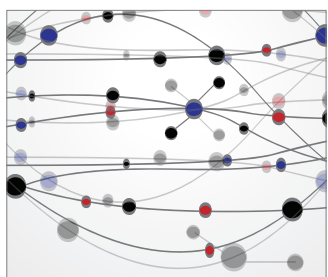

The Scientific World Journal
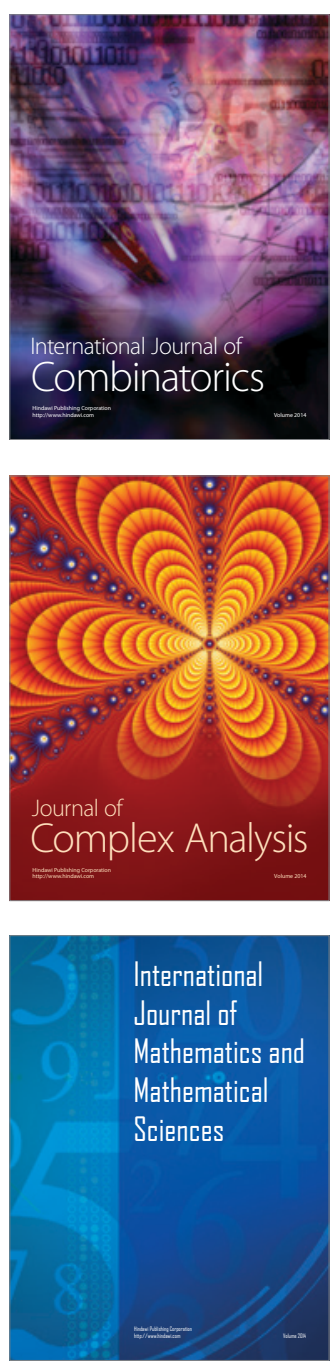
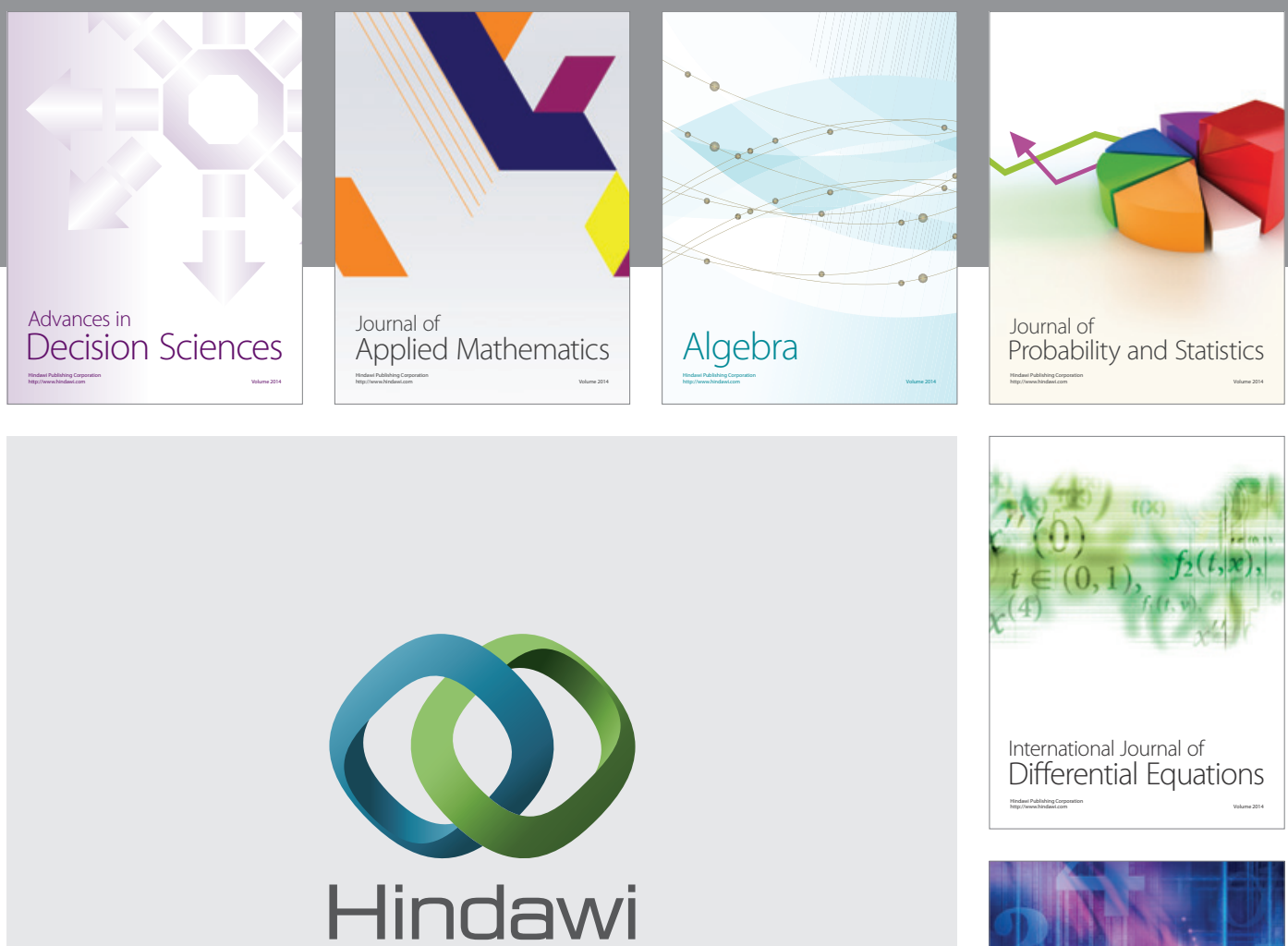

Submit your manuscripts at http://www.hindawi.com
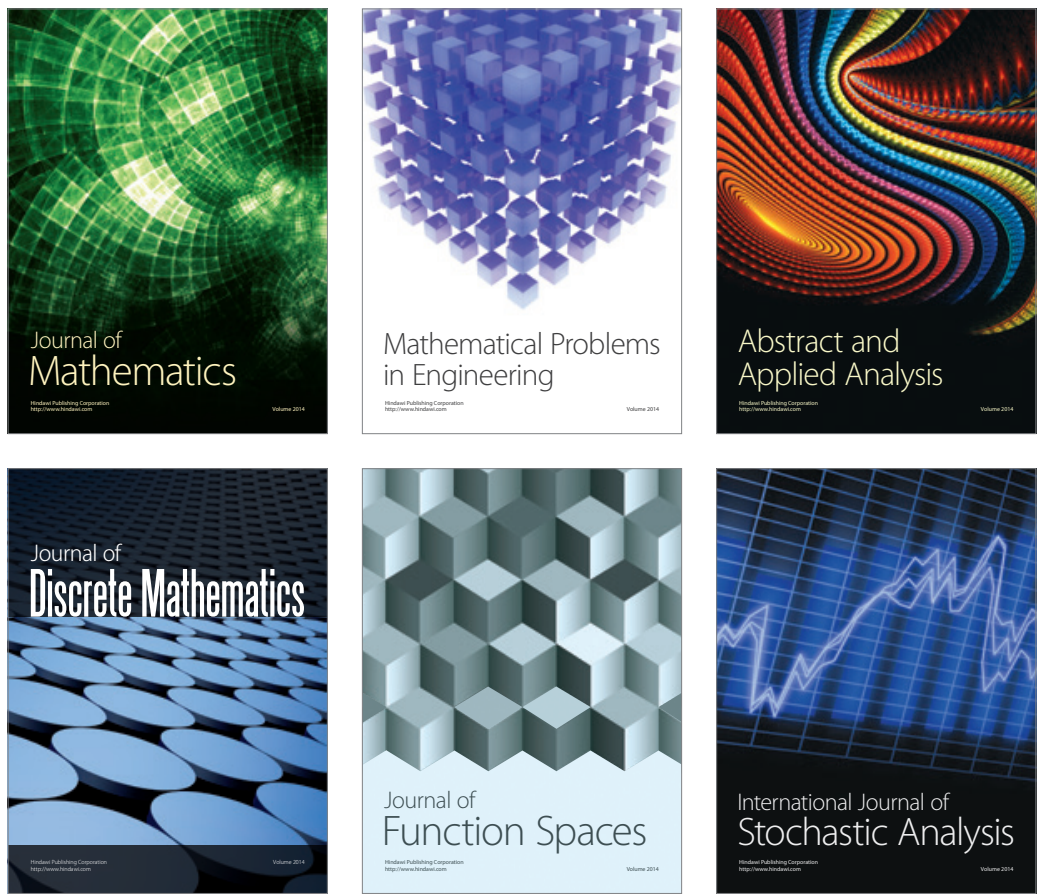

Journal of

Function Spaces

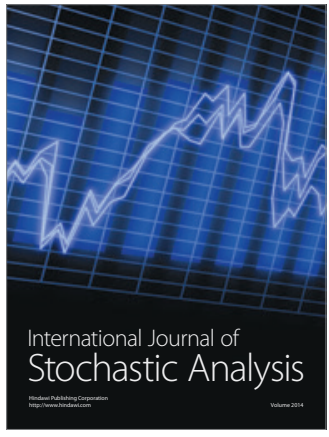

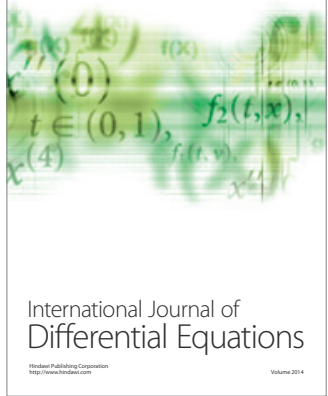
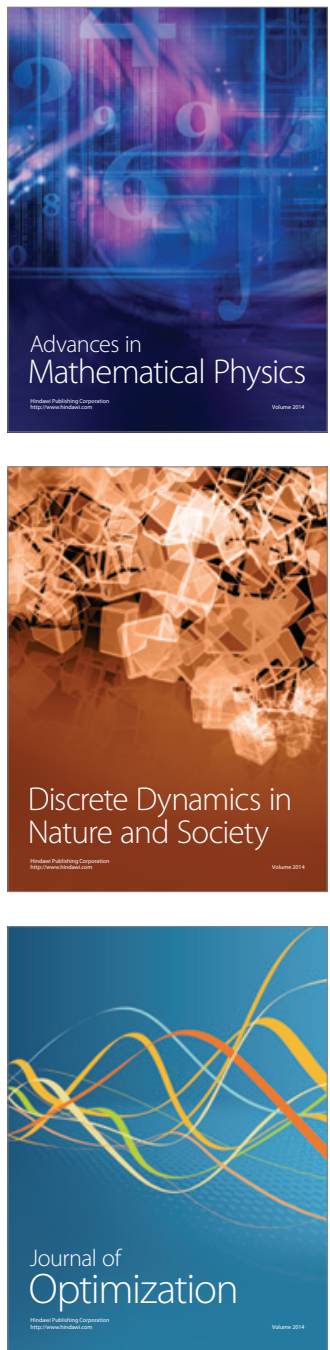